\title{
Dynamic response of tapered optical fiber coated with graphene oxide for detecting aqueous ethanol
}

\begin{abstract}
Typically, evanescent-field optical fiber sensors utilize tapered fiber structure to enhance the evanescent filed interaction with the sensing medium. A nanostructured sensing layer is applied to further enhance the sensitivity of the sensor. This paper investigates the use of tapered fiber sensor coated with graphene oxide (GO) nano-film. Optimization of the GO layer and the tapering parameters were performed and the sensing capability of the device is tested using different concentrations of ethanol. The sensor demonstrates fast response and recovery to aqueous ethanol when interrogated in the visible region using a spectrometer and light source which is 20 seconds and 30 seconds respectively.
\end{abstract}

Keyword: Fiber sensor; Graphene oxide; Tapered fiber 